\title{
INFLUÊNCIA DA PRESSÃO NA MICROESTRUTURA E DUREZA DA LIGA ALSI1MG0.5MN OBTIDA PELO PROCESSO SQUEEZE CASTING *
}

Raelly Laurinda Araujo da Silva ${ }^{1}$ Ariely Moreira da Silva ${ }^{2}$ Mara Cleide Nogueira da Silva ${ }^{3}$ Alan Cunha dos Anjos ${ }^{4}$ Camila dos Santos Torres ${ }^{5}$

\section{Resumo}

As ligas de alumínio são utilizadas mundialmente em inúmeras atividades. Com o aumento da competitividade, existe a necessidade de melhoria constante no processo produtivo. Essas melhorias têm como objetivo reduzir custos e melhorar o desempenho dos componentes e têm sido realizadas pela aplicação de novas técnicas e pelo desenvolvimento de novas ligas/composições. O processo squeeze casting é um processo inovador dentro dos processos de fundição, no qual o metal vazado no estado liquido é solidificado sob pressão dentro de uma coquilha metálica fechada por um punção. Neste contexto, o presente trabalho buscou analisar a influência da aplicação de diferentes pressões no processo de squeeze casting na modificação estrutural da liga de alumínio do tipo AlSi1Mg0.5Mn. Os resultados indicam que o aumento da pressão na liga gera uma taxa de transferência de calor mais elevada que reduziu o tempo de solidificação modificando a microestrutura.

Palavras-chave: Squeeze Casting; Solidificação; Ligas de Alumínio.

\section{INFLUENCE OF PRESSURE ON THE MICROSTRUCTURE AND HARDNESS OF ALSI1MG0.5MN ALLOY PRODUCED BY SQUEEZE CASTING}

\section{Abstract}

Aluminum alloys are used worldwide in numerous activities. With the increase of competitiveness, there is a need for constant improvement in the production process. These improvements aim to reduce costs and improve component performance and have been realized by the application of new techniques and the development of new alloys/compositions. The squeeze casting process is an innovative process within the casting processes in which the liquid metal cast is solidified under pressure into a metal shell closed by a punch. In this context, the present work aimed to analyze the influence of the application of different pressures in the squeeze casting process on the structural modification of AISi1Mg0.5Mn aluminum alloy. The results indicate that the increase in pressure in the alloy results in a higher heat transfer rate which reduced the time of solidification and modifying the microstructure.

Keywords: Squeeze Casting; Solidification; Aluminum Alloys.

1 Eng. de Materiais, graduando, Faculdade de Engenharia de Materiais, UNIFESSPA, Marabá, PA, Brasil.

2 Eng. de Materiais, graduando, Faculdade de Engenharia de Materiais, UNIFESSPA, Marabá, PA, Brasil.

3 Eng. de Materiais, graduando, Faculdade de Engenharia de Materiais, UNIFESSPA, Marabá, PA, Brasil.

4 Eng. de Materiais, graduando, Faculdade de Engenharia de Materiais, UNIFESSPA, Marabá, PA, Brasil.

5 Eng. Metalúrgica, Dra. Professora, Faculdade de Engenharia de Materiais, UNIFESSPA, Marabá, $P A$, Brasil. 


\section{INTRODUÇÃO}

A procura por produtos em alumínio tem aumentado nos últimos anos. Propriedades como densidade, resistência à corrosão, ductilidade e condutividade, fazem do alumínio um material com inúmeras aplicações [1]. As primeiras aplicações comerciais de alumínio foram itens como quadros de espelho, números de casas e bandejas. Com o tempo, o alumínio cresceu em diversidade de aplicações na medida em que praticamente todos os aspectos da vida moderna foram afetados direta ou indiretamente pelo seu uso. Usos típicos incluem estruturas extrudadas, veículos rodoviários, material ferroviário, tubos e tubulações para transportar água, óleo ou gasolina [2].

Apesar de o alumínio puro apresentar baixa resistência mecânica, as suas ligas apresentam uma combinação de propriedades que contribuem para a sua crescente utilização. O silício é na maioria das ligas de alumínio o principal elemento de liga. O sistema alumínio-silício forma um eutético simples limitado pela solubilização no estado sólido dos dois elementos como pode ser observado no diagrama binário na Figura 1. O silício melhora a fluidez do banho metálico, reduzindo os riscos de ocorrência de fissuras a quente. A dureza da liga aumenta com o aumento do teor de silício. Contudo, esse aumento reduz a sua ductilidade, bem como usinabilidade [3].

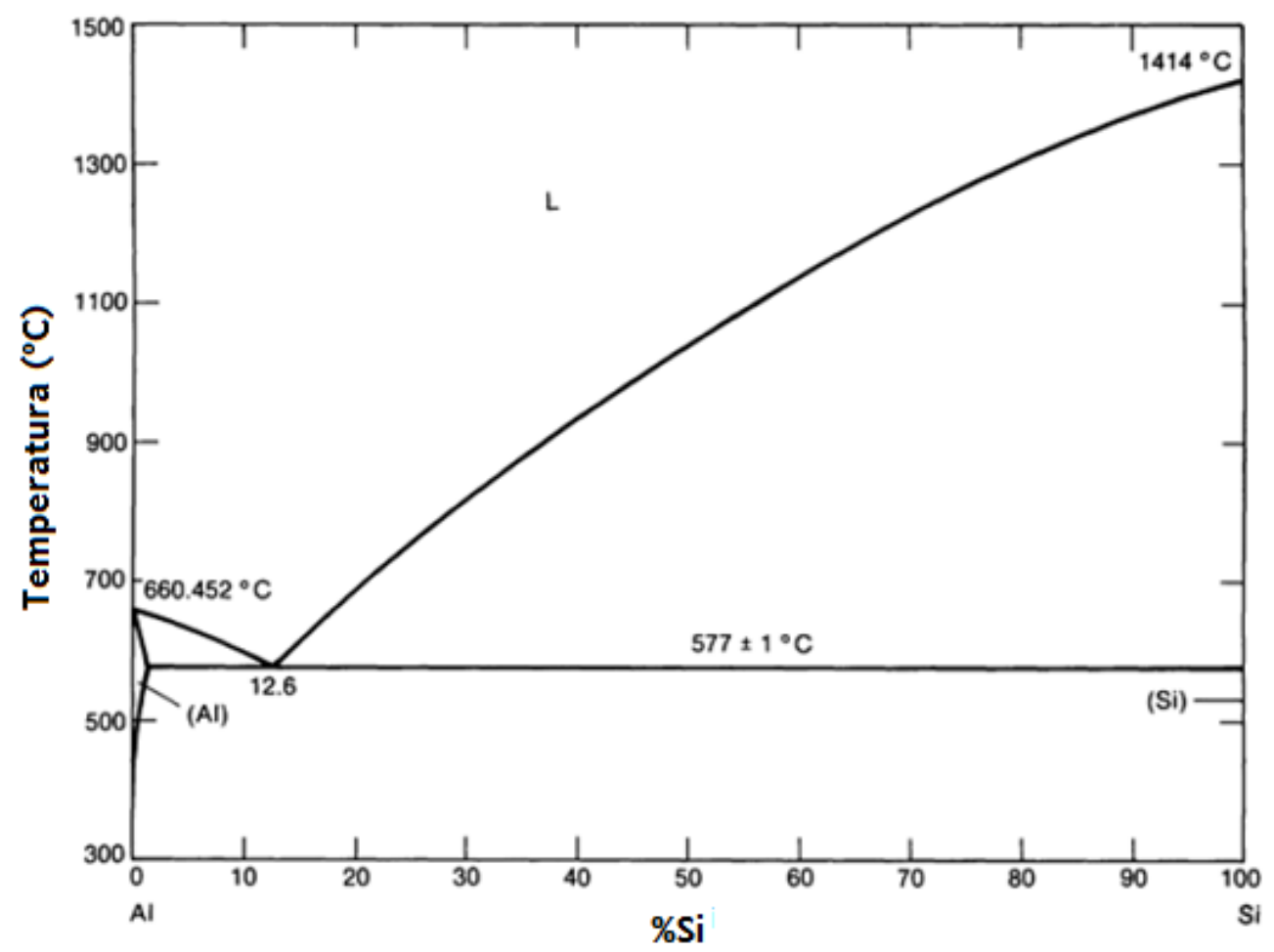

Figura 1: Diagrama de fase do sistema Al-Si. Adaptado de [4].

O alumínio pode ser fundido por essencialmente todos os processos existentes, incluindo fundição sob pressão e molde permanente. As variações importantes incluem moldagem em casca (shell molding) e processos derivados como squeeze casting, injeção de alta pressão a vácuo e fundição semi sólida baseada em princípios de reofundição/ tixofundição. 
Squeeze casting é um processo pelo qual o metal fundido solidifica sob pressão aplicada que é mantida até o final da solidificação. O metal fundido solidifica dentro de uma matriz fechada, posicionada entre as placas de uma prensa hidráulica, a pressão aplicada e o contato do metal com a superfície da matriz melhoram a transferência de calor e inibem a precipitação de hidrogênio e a formação de vazios de contração [5].

A técnica de squeeze casting permite o processamento de ligas com uma estreita faixa de solidificação, que são muito difíceis de obter por outros processos de fundição. O baixo nível de porosidade das peças fundidas por este processo é favorável para ligas que são passíveis de tratamento térmico de envelhecimento [6].

Neste estudo foram analisadas as modificações na microestrutura, dureza e densidade impostas pelas diferentes pressões aplicadas no processo de squeeze casting na liga de alumínio do tipo AlSi1Mg0.5Mn, de forma a relacionar as consequências atribuídas no material. Os resultados indicam que a pressão aplicada é benéfica, permitindo a obtenção de materiais basicamente livres de porosidades com pressões relativamente baixas.

\section{MATERIAIS E MÉTODOS}

Os materiais utilizados neste trabalho foram lingotes da liga de alumínio do tipo AISi1Mg0.5Mn. Esta liga é designada pela Aluminum Association como uma liga da série 6351. Na Tabela 1 é apresentada a composição química teórica da liga utilizada.

Tabela 1. Composição da liga 6351 [7]

\begin{tabular}{ccccccccc}
\hline Designação & \multicolumn{10}{c}{ Composição, \% peso } \\
\hline $\begin{array}{c}\text { Aluminum } \\
\text { Association }\end{array}$ & $\mathrm{Si}$ & $\mathrm{Fe}$ & $\mathrm{Cu}$ & $\mathrm{Mn}$ & $\mathrm{Mg}$ & $\mathrm{Zn}$ & $\mathrm{Ti}$ & Mínimo Al \\
\hline 6351 & $0,7-1,3$ & 0,5 & 0,1 & $0,4-0,8$ & $0,4-$ & 0,2 & 0,2 & Restante \\
& & máx & máx & & 0,8 & máx & & \\
\hline
\end{tabular}

A fusão da liga utilizada foi efetuada em forno Mufla, marca Jung e cadinho de carbeto de silício (SiC), com capacidade de $500 \mathrm{~mL}$. Após a temperatura atingir a temperatura de vazamento correspondente a $7 \%$ de superaquecimento a liga foi vazada em lingoteira de aço, onde foi aplicada a pressão com uma prensa hidrostática semi-automática. As pressões de trabalho utilizadas foram: pressão ambiente, $50 \mathrm{MPa}, 100 \mathrm{MPa}$ e 150MPa.

Para avaliar as modificações resultantes das diferentes pressões utilizou-se metalografia como método de análise da microestrutura da liga. Para revelar a microestrutura foi realizado ataque químico com solução Keller.

Para a caracterização microestrutural das amostras obtidas foi utilizado microscópio ótico marca Zeiss Axioscop 40. Medidas de densidade pelo método de Arquimedes e ensaio de dureza Rockwell $F$ em durômetro da marca Pantec foram realizados nas amostras.

\section{RESULTADOS E DISCUSSÃO}

\subsection{Caracterização microestrutural}


As diferentes velocidades de resfriamento que se atingem com as diferentes pressões aplicadas no processo de squeeze casting conduzem a uma estrutura de grão diferente, como é possível observar na Figura 2. A microestrutura da liga é resultante da composição química da mesma e do processo de fundição.
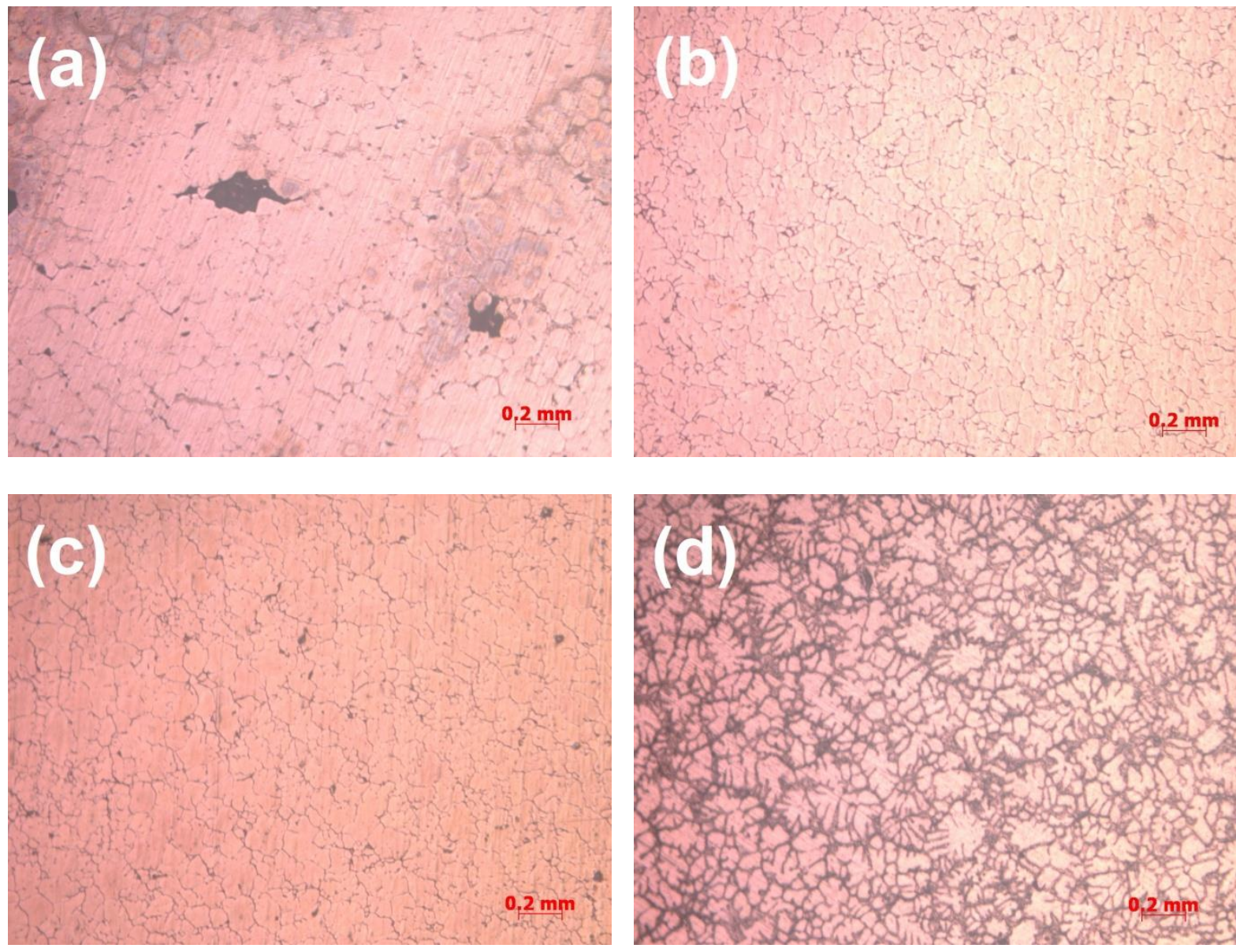

Figura 2. Microestrutura das amostras de AISi1Mg0.5Mn nas pressões de (a) ambiente, (b) $50 \mathrm{MPa}$, (c) $100 \mathrm{MPa}$ e (d) $150 \mathrm{MPa}$. Ataque Keller. Aumento 50X.

$\mathrm{Na}$ amostra obtida em pressão ambiente (Figura 2a) é possível observar um tamanho de grão mais grosseiro, devido à taxa de resfriamento inferior. É também evidente $\mathrm{o}$ aparecimento de poros, provavelmente devido à presença de gases que ficaram retidos no material. A amostra obtida com pressão de $150 \mathrm{MPa}$ (Figura 2d) apresenta microestrutura na qual é dominante a forma dendrítica. Quando a taxa de solidificação é maior o tamanho de grão é mais fino e as dendritas são de menores dimensões $[7,9,10]$.

Devido à pressão, a porosidade diminui e o refinamento microestrutural é conseguido numa extensão muito maior do que na fundição sem aplicação de pressão. $O$ refinamento e a integridade da microestrutura possibilitados pelo squeeze casting permitem ainda a realização de operações secundárias, incluindo soldagem e tratamento térmico [8]. 


\subsection{Dureza e Densidade}

As propriedades mecânicas dos materiais estão diretamente ligadas à sua microestrutura [8, 9]. A Figura 3 apresenta os valores de dureza Rockell $F$ e densidade relativa da liga após o processo de squeeze casting.

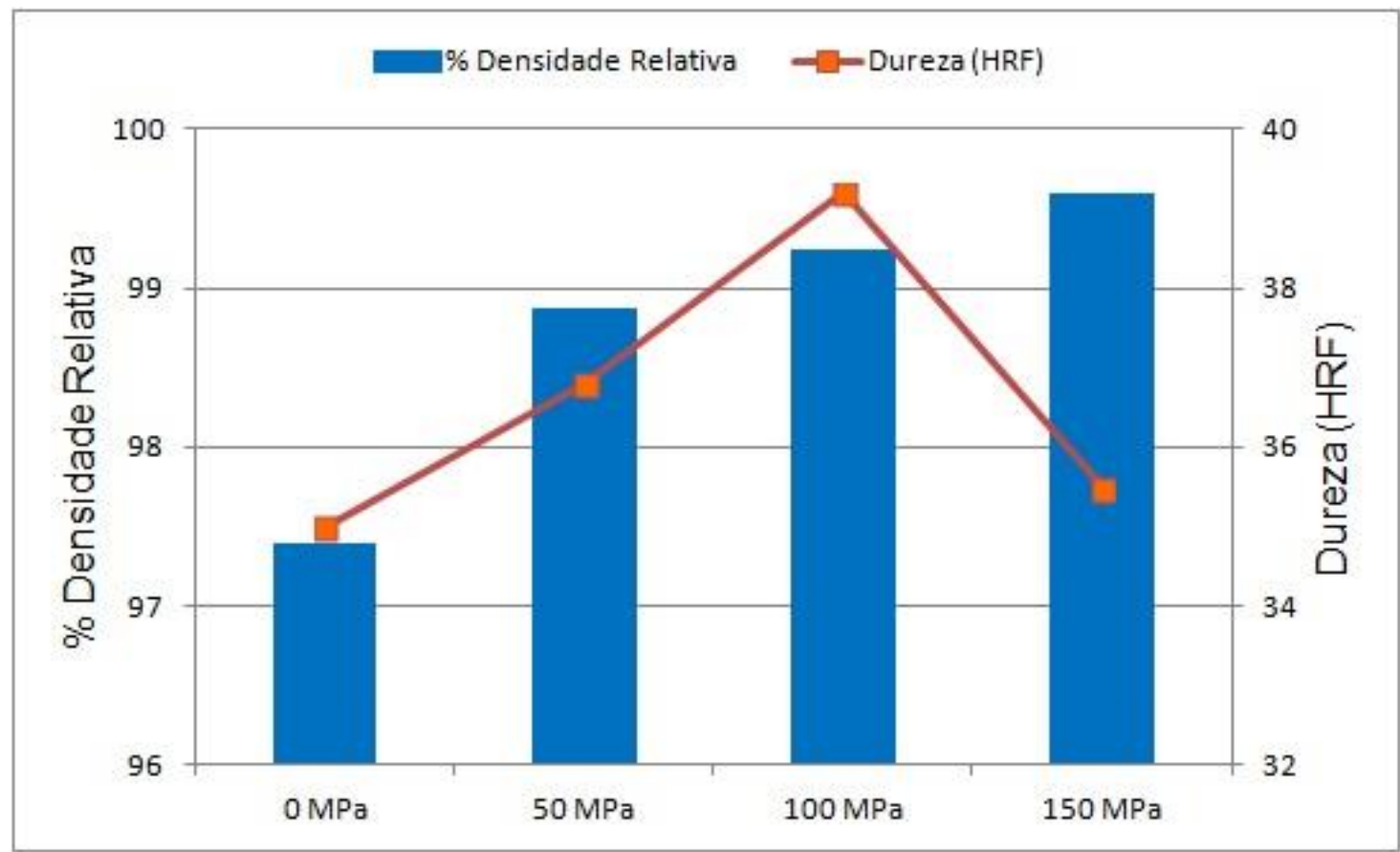

Figura 3. Dureza Rockwell F e densidade relativa em função da pressão aplicada.

Os resultados mostraram um aumento nos valores de dureza da liga obtida em pressão de $50 \mathrm{MPa}$ e $100 \mathrm{MPa}$ em relação a amostra obtida em pressão ambiente. No caso da amostra obtida em pressão de $150 \mathrm{MPa}$, a diferença foi menor quando comparada com a amostra obtida em pressão ambiente.

Quanto menor o tamanho de grão, maior é a quantidade de contornos de grãos, mais resistente é o material devido à dificuldade de movimentação das discordâncias. É importante esclarecer que os valores de dureza permitem uma previsão da resistência mecânica do material, mas não da sua ductilidade, que é grandemente afetada pela morfologia dos grãos e pela presença de intermetálicos.

Pode-se observar que a densidade relativa foi aumentada até a amostra obtida em pressão de $150 \mathrm{MPa}$, alcançando 99,6\% da densidade teórica. A maior densidade para a amostra de $150 \mathrm{MPa}$, esta de acordo com a micrografia apresentada na Figura 2(d) que apresentou uma microestrutura mais homogênea e basicamente isenta de poros.

\section{CONCLUSÃO}

A aplicação de pressão no processo de squeeze casting causa uma mudança na microestrutura da liga, é evidente a diminuição do grão como aumento da pressão. A amostra obtida em pressão ambiente apresentou uma estrutura celular enquanto que a amostra com pressão de $150 \mathrm{MPa}$ apresentou estrutura dendrítica predominante. As amostras obtidas nas pressões de $50 \mathrm{MPa}$ e $100 \mathrm{MPa}$ apresentaram estruturas de transição de celular/dentrítica. 
A dureza da liga apresentou maior valor para a amostra obtida na pressão de $100 \mathrm{MPa}$, são necessários ensaios adicionais para ajudar na definição da evolução deste parâmetro. Os valores de densidade relativa foram maiores para a amostra obtida em maior pressão, resultando em um material praticamente livre de porosidade.

\section{Agradecimentos}

Os autores agradecem ao apoio do CNPq e da Pró-reitoria de Pós-graduação, Pesquisa e Inovação Tecnológica - PROPIT.

\section{REFERÊNCIAS}

1 Oliveira GRG. Tratamento térmico de uma liga Al-Si-Mg-Mn. [dissertação de mestrado]. Portugal: Faculdade de Engenharia da Universidade do Porto; 2012.

2 ASM HandBook Vol 2. Properties and Selection: Nonferrous Alloys and SpecialPurpose Materials. ASM International; 1991.

3 Brown JR. Foseco Non-Ferrous Foundryman's Handbook. 11th ed. Oxford: Butterworth Heinemann; 1999.

4 Massalski TB, Murray JL, Bennett LH, Baker H. Binary Alloy Phase Diagrams. 2nd ed. ASM International; 1990.

5 Kaufman JG, Rooy, EL. Aluminum Alloy Castings: Properties, Processes, and Applications. ASM International; 2004.

6 Friedrich HE, Mordike BL. Magnesium Technology: Metallurgy, Design Data, Automotive Applications. Springer Science \& Business Media; 2006.

$7 \quad$ ASM HandBook Vol 15. Casting. 9th ed. ASM International; 1998.

8 Smith WF. Structure and Properties of Engineering Alloy. 2nd ed. McGraw-Hil; 1993

9 Garcia A. Solidificação: fundamentos e aplicações. São Paulo: Editora Unicamp; 2011.

10 Rajagopal S, Altergott WH. Quality Control in Squeeze Casting of Aluminum, AFS Transactions, 1985;93:145-154. 